\title{
Attosecond Streaking in the Water Window: A New Regime of Attosecond Pulse Characterization
}

\author{
Seth L. Cousin, ${ }^{1}$ Nicola Di Palo, ${ }^{1}$ Bárbara Buades, ${ }^{1}$ Stephan M. Teichmann, ${ }^{1}$ M. Reduzzi, ${ }^{2,3}$ M. Devetta, ${ }^{3}$ \\ A. Kheifets, ${ }^{4}$ G. Sansone, ${ }^{2,3,5}$ and Jens Biegert ${ }^{1,6, *}$ \\ ${ }^{1}$ ICFO-Institut de Ciencies Fotoniques, The Barcelona Institute of Science and Technology, \\ 08860 Castelldefels (Barcelona), Spain \\ ${ }^{2}$ Dipartimento di Fisica, Politecnico Milano, Piazza Leonardo da Vinci 32, 20133 Milano, Italy \\ ${ }^{3}$ Instituto di Fotonica e Nanotecnologie (CNR-IFN), Politecnico Milano, \\ Piazza Leonardo da Vinci 32, 20133 Milano, Italy \\ ${ }^{4}$ Research School of Physics and Engineering, The Australian University, Canberra, ACT 2601, Australia \\ ${ }^{5}$ Institute of Physics, University of Freiburg, Hermann-Herder-Strasse 3, 79104 Freiburg, Germany \\ ${ }^{6}$ ICREA, Pg. Lluís Companys 23, 08010 Barcelona, Spain \\ (Received 24 May 2017; published 2 November 2017)
}

\begin{abstract}
We report on the first streaking measurement of water-window attosecond pulses generated via highharmonic generation, driven by sub-2-cycle, carrier-to-envelope-phase-stable, 1850-nm laser pulses. Both the central photon energy and the energy bandwidth far exceed what has been demonstrated thus far, warranting the investigation of the attosecond streaking technique for the soft-x-ray regime and the limits of the FROGCRAB retrieval algorithm under such conditions. We also discuss the problem of attochirp compensation and issues regarding much lower photoionization cross sections compared with the extreme ultraviolet in addition to the fact that several shells of target gases are accessed simultaneously. Based on our investigation, we caution that the vastly different conditions in the soft-x-ray regime warrant a diligent examination of the fidelity of the measurement and the retrieval procedure.
\end{abstract}

DOI: 10.1103/PhysRevX.7.041030

\section{INTRODUCTION}

Excitation, scattering, and relaxation of electrons are core phenomena that occur during the interaction between light and matter. It is essential to study the temporal dynamics of electrons [1] since their behavior determines how a chemical bond forms or breaks, their confinement and binding determines how energy flows, and these phenomena govern the efficiency of modern organic solar cells or the speed of electronics, all alike. Furthering our understanding therefore requires the capability to localize an excitation in a molecule, or a material, and to follow the flow of energy with attosecond temporal resolution. Spectacular progress has been made in attoscience research since the first real-time observation of the femtosecond lifetime of the $M$-shell vacancy in krypton in 2002 [2]. Electron tunneling [3], time delays in photoemission [4,5], an atom's response during photoabsorption [6], electron localization during molecular dissociation [7], ultrafast charge transfer in biomolecules [8], phase transitions [9], and electron dynamics in condensed matter [10] were

\section{*Jens.Biegert@icfo.eu}

Published by the American Physical Society under the terms of the Creative Commons Attribution 4.0 International license. Further distribution of this work must maintain attribution to the author(s) and the published article's title, journal citation, and DOI.
Subject Areas: Atomic and Molecular Physics

investigated. However, despite these achievements, isolated attosecond pulses [11-15] were generated only in the extreme ultraviolet range (EUV or XUV), i.e., at photon energies lower than $124 \mathrm{eV} \mathrm{[16],} \mathrm{thereby} \mathrm{confining} \mathrm{inves-}$ tigations largely to valence electron dynamics.

Isolated attosecond pulses at higher photon energies than the XUV (10-124 eV), in the soft-X-ray range (SXR), permit localizing the initial excitation step through ionization of a specific core level of a distinct target atom. This fingerprinting capability is essential for localization of the flow of energy and excitation since it permits interrogating the entire electronic structure of an atom, molecule, or solid with ultrafast temporal resolution and element selectivity. Such soft-x-ray attosecond pulses will give access to a plethora of fundamental processes like intra-atomic energy transfer [17] and its range [18], charge-induced structural rearrangement [19], change in chemical reactivity [20], photodamage of organic materials [21], emergence of reflectivity [22], and carrier scattering [23], recombination [24], and exciton dynamics [25], just to name a few. Attosecond pulses in the SXR spectral range will also give access to fundamental ultrafast electron transfer between adsorbates and surfaces which have been accessible so far only by alternative approaches such as core-hole spectroscopy [26]. Another enticing possibility with attosecond soft-x-ray pulses is the realization of ultrafast electron diffraction from within the molecule itself [27]. This concept requires the measurement of the electron 
interference pattern arising from an initially well-defined and confined electronic wave packet inside the molecule, thus calling for an initially localized ionization process. Soft-x-ray pulses with duration at, or below, the atomic unit of time (24 as) would provide a universal tool to access the time scales of exchange and correlation which characterize the universal response of electronic systems after the sudden removal of an electron and which occur on the characteristic time scale of less than 50 as [28]. Such pulses will also enable us, for the first time, to time resolve the few attosecond dynamics inside tailored materials, whose subfemtosecond dynamics are largely unexplored [29].

Here, we present the generation of attosecond-duration pulses with photon energy in the soft-x-ray range up to $350 \mathrm{eV}$. This photon energy is sufficiently high to fully cover the $K$-shell absorption edge of carbon at $284 \mathrm{eV}$ for spectroscopic applications [30-32]. We demonstrate the first attosecond streaking measurement in the soft-x-ray water-window regime and confirm an upper limit of the duration of the isolated pulse of 322 as. These results herald a new era of attosecond science in which subfemtosecond temporal resolution is paired with element selectivity by reaching the fundamental absorption edges of important constituents of matter such as carbon.

\section{FEMTOSECOND AND ATTOSECOND PULSE GENERATION AND CHARACTERIZATION}

With the initial generation of ultrashort pulses (defined here as pulses that are too short to be measured directly using modern detectors and electronics), an entire field of optics has arisen to address the challenge of characterizing them. Frequency-resolved optical gating (FROG) [33] and spectral phase interferometry for direct electric-field reconstruction (SPIDER) [34] solved this challenge, by using replicas of the pulse itself combined with nonlinear interactions to either reduce the problem to a solvable phase retrieval issue in the case of FROG, or to directly extract the phase through Fourier analysis of the nonlinear signals recorded in the case of SPIDER. Because of the relationship between spectral bandwidth and pulse duration, further significant reduction of pulse durations to the subfemtosecond regime is not possible directly from laser media; however, through novel pulse compression schemes, such as filamentation and hollow-core fiber pulse compression [35-40], pulse durations continued to decrease to the single-cycle limit. Subcycle pulses have also been synthesized [41] in schemes where discrete broad spectra can be combined coherently. Another approach to the generation of subfemtosecond radiation comes fortuitously and intrinsically from the process of high-harmonic generation (HHG) [42]. HHG results from the interaction of an ultrashort, intense laser pulse with typically a gas-phase medium. The laser pulse first facilitates the tunnel ionization of an electron from the gas atom, whereafter it accelerates the electron initially away and then back to the parent ion [43]. In the event of recombination with the ion, the kinetic energy obtained during its excursion is released in the form of high-harmonic radiation. The energy range and cutoff that is achieved during HHG is dependent predominantly on the wavelength of the driving laser. In the past few years, there has been a significant push to generate bright HHG radiation at higher photon energies by driving the process with longer wavelength sources. The HHG process repeats every half-cycle of the driving laser, which at $800 \mathrm{~nm}$ corresponds to $2.7 \mathrm{fs}$ per cycle. Not all electrons ionize or recombine at the same time, which in turn implies that the high-harmonic photons are also born at different times. A pulse in which different wavelengths are temporally dispersed is by definition a chirped pulse and in this specific context the dispersion is called attochirp. Even in the case of maximum attochirp, the subcycle nature of the process guarantees subcycle high-harmonic pulse durations. Another consequence of the half-cycle repetition frequency of the process is that every half-cycle with the intensity to initiate the process can result in a burst of this attochirped radiation, so for a multicycle pulse, i.e., $30 \mathrm{fs}$ at $800 \mathrm{~nm}$, multiple attosecond bursts will be present, which results in a train of attosecond pulses. The obvious path to an isolated attosecond pulse is then to ensure that only one half-cycle dominates the HHG process, which can be achieved through various gating techniques [12-15,44]. A fortuitous temporal gating arises as a consequence of successful phase matching of the HHG in particular conditions [42] especially relevant to this work. These gating techniques, in combination with control of the carrier-to-envelope phase (CEP) of the laser pulses, can be used to select the pulse's electric-field shape in which predominantly only one half-cycle dominates and only one repeatable isolated attosecond pulse is generated.

Extending existing pulse characterization techniques to attosecond pulses is not as straightforward as using the same nonlinear interactions as used for few femtosecond pulses; i.e., second-harmonic generation in bulk is not possible at the short $\mathrm{x}$-ray wavelengths arising from HHG. What can be exploited, however, is the perturbation of electrons (ionized from atoms irradiated by the $\mathrm{x}$ rays) by a phase-locked copy of the pulse that generated the harmonic radiation. Photoelectron spectrograms generated by both attosecond pulse trains and isolated pulses can be evaluated using different techniques, namely, attosecond streaking [45] for isolated attosecond pulses and reconstruction of attosecond beating by interference of two-photon transitions (RABBIT) [46] to yield the pulse duration of the harmonic radiation.

The experimental basis of the attosecond streaking measurement is an interferometer in which one arm carries the x-ray radiation and the other arm carries a fraction of the fundamental radiation that was used to generate the $\mathrm{x}$-ray radiation. After the arms of the interferometer are recombined, the combined components irradiate a gas 
target. The $\mathrm{x}$ rays have sufficient energy to photoionize the gas target, whereas the weaker fundamental radiation just has the energy to perturb the ejected photoelectrons. By measuring the time of flight of the photoelectrons as a function of delay between the two arms of the interferometer, the momentum shift of the photoelectrons is recorded in the form of a streaking spectrogram. Encoded in the spectrogram is the phase of the electron wave packet, which is assumed to be identical to the $\mathrm{x}$-ray photon wave packet. An iterative phase retrieval algorithm, such as frequencyresolved optical gating for complete reconstruction of attosecond bursts (FROGCRAB) [47] can be used to reconstruct the full electric field of the x-ray pulse and hence its pulse duration.

\section{ATTOSECOND STREAKING}

To date, virtually all attosecond streaking measurements reconstructed by the FROGCRAB algorithm have been performed on attosecond pulses that have been generated by HHG, driven with 800-nm titanium sapphire (Ti:sapphire) lasers $[3,4,11-13,48-50]$. Recent research into using these algorithms suggests that care needs to be taken if very accurate reconstruction is essential and that other algorithms may perform better [51-53]. Because of the scaling of the HHG cutoff energy [54], the spectra supporting these pulses has been restricted to XUV photon energies and bandwidths well below $50 \mathrm{eV}$. To access higher photon energies efficiently, the only way forward is to use longer wavelength radiation to drive HHG. Technically, this means either the addition of wavelength conversion schemes after the Ti:sapphire laser system or a complete new laser architecture, such as optical parametric amplification (OPA) or optical parametric chirped pulse amplification. Both of these options pose significant technical challenges. There are only a handful of systems worldwide capable of supplying the stable and extreme radiation needed to generate repeatable isolated attosecond pulses in the water-window range, i.e., central wavelengths larger than 800-nm, few-cycle, CEP-controlled laser pulses with sufficient energy to facilitate phase-matched HHG.

Because of the wavelength scaling of the single atom response $\left(\lambda^{-5}\right.$ to $\left.\lambda^{-9}\right)$ [55,56], phase-matched HHG using longer wavelength radiation relies on multiatmosphere gas pressure to balance the phase mismatch between the fundamental driving laser and the generated harmonics [57]. These multiatmosphere gas pressures are achieved either via capillary waveguide targets or through the correct engineering of vacuum pumping systems to facilitate an easy-to-align free-space target. Again, there are only a handful of systems reported capable of achieving these environments.

In the case of XUV attosecond pulses, photoionization occurs from the valence or inner valence shells, while for soft-x-ray (for example, in the water window 283-543 eV) photoelectrons, core-shell electrons are preferentially released in the continuum. Even though gaining access to these levels is interesting for the purpose of electronic processes triggered by a core vacancy, it can be a hindrance for the implementation of streaking measurements, due to the photoionization cross sections for core levels, which are significantly lower than for valence shells (see Fig. 1). This effect, combined with the lower flux of high-photon energy in the soft $\mathrm{x}$ ray, potentially makes the acquisition of a streaking spectrogram a lengthy process.

The broad spectra associated with isolated attosecond pulses in the water window span over multiple core shells of the typical gases used. The overlap of streaking traces from two different shells heavily complicates interpretation of the streaking spectrogram. Using helium as the target gas can mitigate this issue; however, the ionization cross section is extremely low. The $3 d$ shell of krypton stands out as a viable option for water-window streaking as it has a relatively high cross section compared to krypton's other core shells and has a relatively flat response over a broad bandwidth.
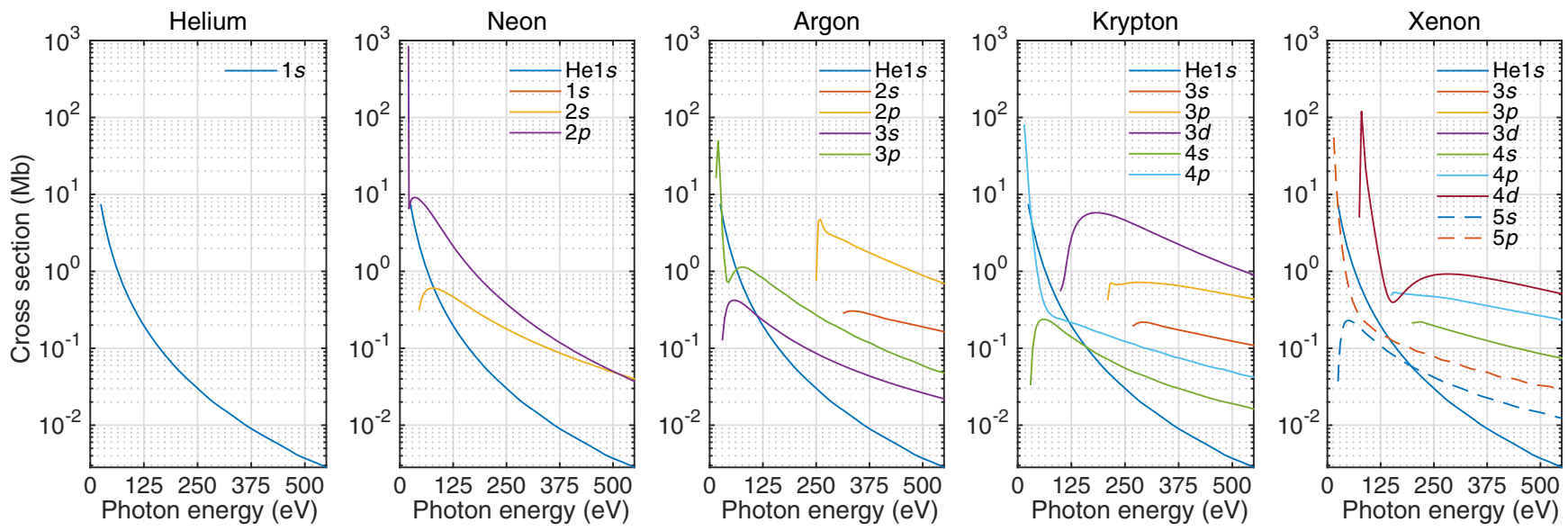

FIG. 1. Photoionization cross sections [58] for the typically used target gases in attosecond streaking experiments. Helium $1 s$ cross section is included in dark blue in each plot as a point of reference. 


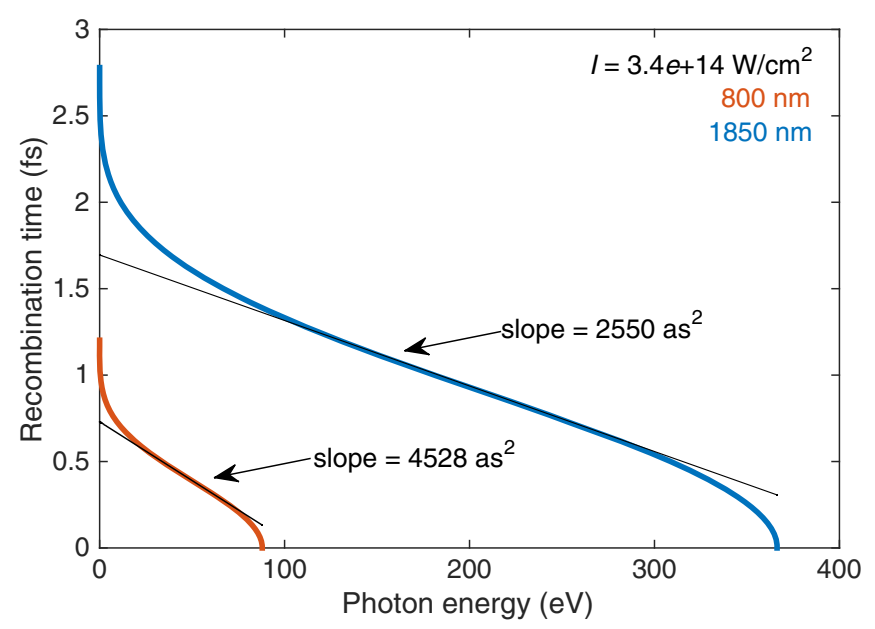

FIG. 2. Attochirp as a function of driving wavelength, $800 \mathrm{~nm}$ (red curve) and $1850 \mathrm{~nm}$ (blue curve).

\section{ATTOCHIRP COMPENSATION}

Attochirp is intrinsic to the HHG process, and in order to compensate for the chirp, either a suitable postcompression technique or a method [59] to mitigate attochirp is needed. Short trajectory harmonics have traditionally been selected via placement of the target with respect to the focus. Attosecond pulses emanating from short trajectory harmonics are positively chirped (low-energy electrons recombine before the high-energy electrons). Negative chirp can be introduced by the thin metal foil filter [60] used to reject the fundamental laser from the copropagating XUV pulse. One of the benefits of driving $\mathrm{HHG}$ with long wavelength drivers is that the magnitude of the intrinsic attochirp scales inversely with wavelength [61] and intensity [62]. Figure 2 highlights this, showing the calculated slope of recombination for both $800-$ and 1850-nm driven harmonics. It has also been proposed that atomic gas can be used as an attochirp compressor [63]; however, unlike in the XUV regime, beyond $200 \mathrm{eV}$ chirp compensation is hard to come by.

TABLE I. GDD and transmission of gases and solids for attochirp compensation.

\begin{tabular}{|c|c|c|c|c|}
\hline \multirow{2}{*}{$\begin{array}{l}\text { Material } \\
\text { Solid }(100 \mathrm{~nm})\end{array}$} & \multicolumn{2}{|c|}{ GDD $\left(\mathrm{as}^{2}\right)$} & \multicolumn{2}{|c|}{ Transmission $(\%)$} \\
\hline & $50 \mathrm{eV}$ & $250 \mathrm{eV}$ & $50 \mathrm{eV}$ & $250 \mathrm{eV}$ \\
\hline Aluminium (Al) & -673 & -7 & 83 & 34 \\
\hline Zirconium (Zr) & -1975 & -18 & 0.6 & 17 \\
\hline Titanium (Ti) & 11633 & -13 & $\cdots$ & 62 \\
\hline Tin $(\mathrm{Sn})$ & 1087 & -13 & 0.09 & 58 \\
\hline Chromium (Cr) & 3114 & -18 & 0.3 & 36 \\
\hline \multicolumn{5}{|c|}{ Gas (1 mm, 100 mbar) } \\
\hline Neon $(\mathrm{Ne})$ & 133 & -4 & 17 & 87 \\
\hline Xenon (Xe) & -1168 & -6 & 72 & 69 \\
\hline Krypton (Kr) & -978 & 5 & 68 & 30 \\
\hline Argon (Ar) & -1083 & 1 & 81 & 31 \\
\hline
\end{tabular}

The group delay dispersion (GDD) of the materials used for compression is far less effective, as shown in Table I, which shows GDD and transmission for different materials at $50 \mathrm{eV}$ and at $250 \mathrm{eV}$. Chirped attosecond pulses with central energies below $100 \mathrm{eV}$ have already been compressed using metallic foils [12]. For a chirped attosecond pulse with a central energy of $250 \mathrm{eV}$, to compensate for $2500 \mathrm{as}^{2}$ of chirp using zirconium, we would need a $\sim 13-\mu \mathrm{m}$ thick foil, or in neon gas we would need $\sim 6$ bar in a $10-\mathrm{mm}$ cell. The transmission through either, however, would be negligible. Attochirp compensation of pulses beyond $200 \mathrm{eV}$ may not be possible using the traditional postcompression schemes, but more novel schemes may be needed.

\section{EXPERIMENTAL SETUP}

To fulfill the stringent requirements of the laser source needed to generate isolated attosecond pulses at highphoton energies, we dedicate extensive time and effort to our light source. The system is based upon a cryogenically cooled, two-stage, Ti:sapphire amplifier delivering stable, robust, $40-\mathrm{fs}, 7-\mathrm{mJ}$ pulses at $1 \mathrm{kHz}$ with immaculate beam quality. These pulses are used to seed a (TOPAS-HE) OPA in which three stages of white-light seeded amplification results in passively CEP-stabilized 45-fs idler pulses at a center wavelength of $1850 \mathrm{~nm}$. A hollow-core fiber pulse compressor is then used to spectrally broaden these pulses to support sub-2-cycle pulse durations, which are compressed in bulk to give 0.4-mJ, 12-fs CEP-stable pulses. An additional slow feedback loop ensures that slow drifts of CEP fluctuations can be mitigated over arbitrary time durations.

These extreme laser pulses enter an attosecond streaking beam line depicted in Fig. 3, where the majority of their energy is devoted to harmonic generation. They are focused down to $54 \mu \mathrm{m}$ to achieve an intensity of $4.3 \times 10^{14} \mathrm{~W} / \mathrm{cm}^{2}$ in a free-space target with an interaction region $<1 \mathrm{~mm}$ long and a backing pressure of 3 bar of neon. (See Ref. [30] for more details regarding HHG, target geometry, and water-window spectroscopy with this source.) Soft-x-ray radiation is generated well into the water-window range resulting in flux of $5.6 \times 10^{5}$ photons/s from 284 to $350 \mathrm{eV}$ on target. The remaining energy is split to be used as the perturbing streaking field in the streaking measurement, ultimately achieving an intensity of $3.2 \times 10^{11} \mathrm{~W} / \mathrm{cm}^{2}$ in the focus.

The $\mathrm{x}$ rays are refocused to a TOF spectrometer using an ion-beam polished, grazing incidence, ellipsoidal x-ray optic, possessing a measured surface roughness of less than $0.5 \mathrm{~nm}$ over the whole surface $\left(260 \times 50 \mathrm{~mm}^{2}\right)$. A gold mirror with a $3-\mathrm{mm}$ through-hole, drilled at $45^{\circ}$, facilitates the recombination and coalignment of the 1850 -nm streaking pulses (reflecting from the front surface) and the $x$ rays, which propagate unabated through the drilled aperture due to their low divergence. This coalignment is made possible under vacuum due to the 


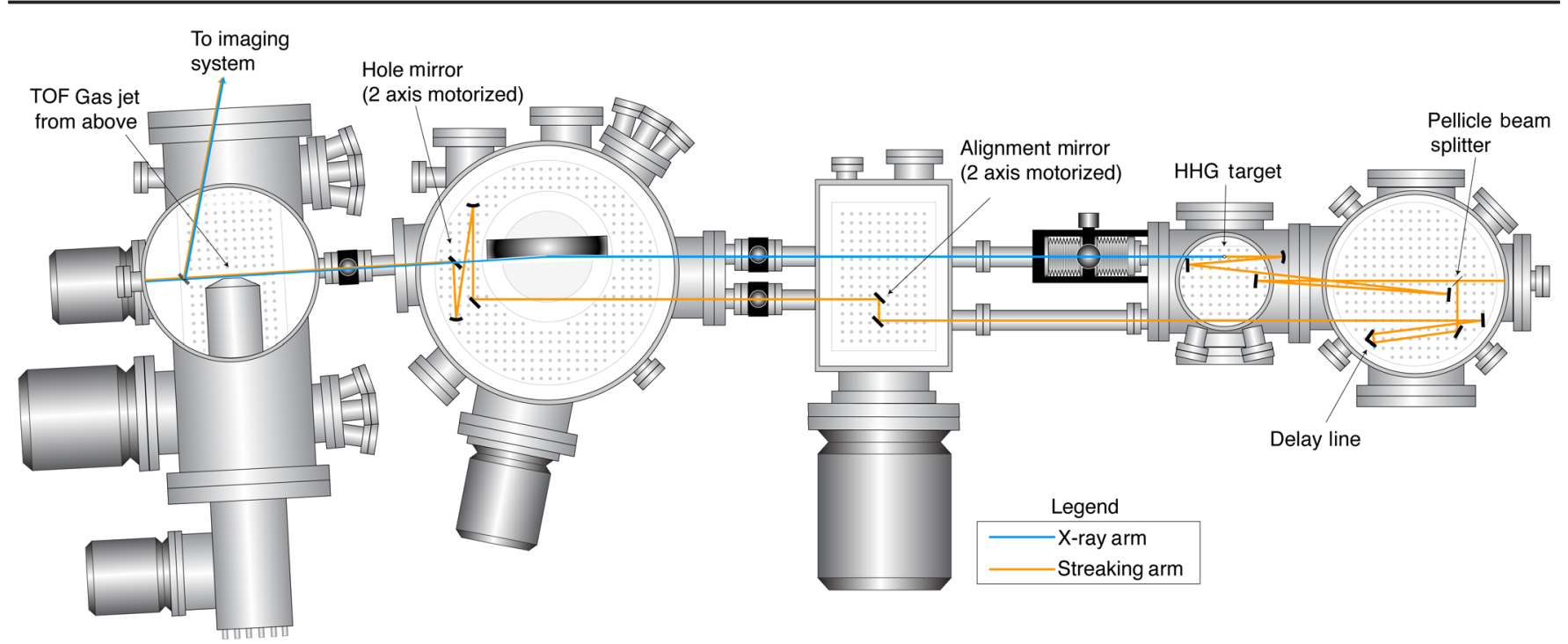

FIG. 3. Schematic layout of the attosecond streaking beam line. Five interconnected vacuum chambers make up the attosecond streaking beam line. Following the beam propagation direction, from right to left, the first two chambers are only rough pumped to facilitate the high gas pressures needed for phase-matched HHG, and the last three chambers are turbopumped to minimize reabsorption of the $\mathrm{x}$ rays and ensure the required pressure environments needed by the diagnostics in the last chamber (left).

use of motorized mirrors for beam steering, as well as an in-vacuum actuator-controlled beam sampling mirror extracting the combined beams to an imaging system. When the mirror is inserted, a beam profiling camera can either image the plane of the hole-drilled mirror, or by translating the camera farther away, a far-field image can be seen. Collinearity is verified when $1850-\mathrm{nm}$ beams from both arms are centered on the hole and remain spatially overlapped in the far field. The TOF spectrometer (Stefan Kaesdorf ETF10) can be operated in either an electron or ion collection mode and incorporates a 461-mm field free drift tube. The spectrometer offers a TOF resolution of $T / D T=100$, which translates to an energy resolution of $E / D E=50$. We discuss the impact of spectral resolution in Sec. VID. An Einzel lens is employed to focus charged particles to a microchannel plate without changing their energies. Optimal focusing of the electrons is achieved when a lens voltage of around 5 times the expected electron energy is applied. Energy acceptance for this lens voltage is approximately Gaussian with a central energy around $200 \mathrm{eV}$ and a FWHM bandwidth of around $150 \mathrm{eV}$. In this configuration, however, the normal acceptance angle of the TOF spectrometer $\left(30^{\circ}\right.$ full cone) is replaced by a spherical acceptance volume with a diameter of $200 \mu \mathrm{m}$. This requires diligent alignment, as it is imperative to coalign the soft $\mathrm{x}$ rays, the streaking IR, and the gas jet all within the small volume.

\section{ATTOSECOND STREAKING IN THE SXR}

Figure 3 depicts the implementation of the attosecond streaking interferometer. Interferometric stability is qualitatively evaluated by monitoring the fringes generated by the interference of 1850-nm radiation in both arms of the interferometer, measured at the location of the TOF gas jet. Without any active stabilization, the fringe stability is excellent, suggesting no need to stabilize the system. The beam line design specifically isolates the chambers from the optical breadboards inside, assisting with the stability. Note that no infrared attenuation filter is used and hence no attochirp compensation is expected. This is done to maximize the flux on target; however, various tests were carried out to affirm that any remnant infrared would not influence the streaking trace. Firstly, the remnant infrared is well below the intensity needed to ionize the krypton TOF gas jet, estimated to be 3 orders of magnitude lower than that of the streaking infrared. Next, even when assuming the remnant $1850-\mathrm{nm}$ radiation is copropagating with the soft $\mathrm{x}$ rays, it would be phase locked with the attosecond burst. In the case that there is significant intensity in the infrared, electrons ionized in the TOF gas jet by the soft $\mathrm{x}$ rays would experience a constant momentum shift, which would manifest in the photoelectron spectra as a constant offset in electron energy. We confirm this is not the case, by examining the photoelectron spectra generated in identical conditions, however, first with a thin filter combination of $200 \mathrm{~nm}$ carbon and $200 \mathrm{~nm}$ chromium blocking the infrared and then with no filter. No shift in electron energy is detected, only a significant attenuation of the signal.

\section{A. Recording the streaking trace-SXR generation in $\mathrm{Ne}$ and detection in $\mathrm{Kr}$}

The TOF spectrometer records the streaking spectrogram as we vary the delay between the two arms of the 


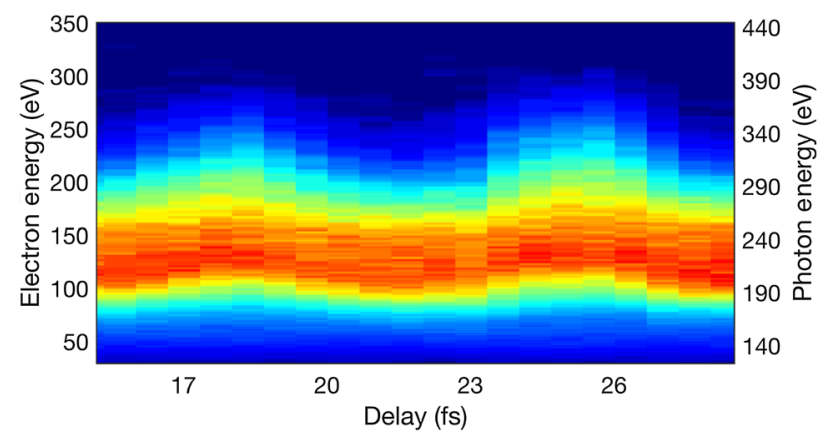

FIG. 4. Streaking trace (linear scale) from the water-window attosecond pulse recorded in $\mathrm{Kr}$.

interferometer. The raw data recorded over a period of $10 \mathrm{~h}$ are shown in Fig. 4, in which TOF spectrometer calibration is applied to convert flight times to electron and photon energies. The electron count rate during the data acquisition is optimized to yield 300 counts/s. Specific attention can be drawn to some of the unprecedented features of this streaking spectrogram, namely, (i) the central electron energy $(150 \mathrm{eV})$ translating to a central photon energy of $\sim 250 \mathrm{eV}$ (ionized from the $3 d$ shell with an ionization potential of $94 \mathrm{eV}$ ) and (ii) the broad bandwidth $(>100 \mathrm{eV}$ ), which supports a pulse duration of 20 as (well below the atomic unit of time). Other features that can be inferred from this raw data before any algorithmic processing is performed, which are discussed in detail through the rest of this article, include (iii) the streaking excursion, albeit on the order of $50 \mathrm{eV}$ is relatively low compared to the bandwidth, (iv) the spectrogram does not exhibit any clear asymmetry on the leading versus the trailing edges of the modulations (asymmetry is visual manifestation of attochirp), implying that we have an unchirped pulse, and (v) there is no indication of multiple "ghosted" phase offset traces.

\section{B. Multiple emissions and multiple shells-XUV generation in $\mathrm{Ar}$ and detection in $\mathbf{K r}$}

In order to investigate the possibility of emission from different shells, we switch from Ne to Ar since it generates much lower photon energies that will access multiple shells in the detection gas Kr. Figure 5 shows streaking spectrograms taken with identical laser parameters but for HHG in argon (at 1 bar), thus resulting in radiation with a $100-\mathrm{eV}$ central energy. The figure displays traces for two values of CEP, with clear evidence of multiple attosecond pulses versus an isolated pulse. A ghosted trace that is a half-cycle out of phase with the primary trace could predominantly come from two sources: (1) a second attosecond burst generated by a preceding or proceeding, lower intensity, half-cycle, or (2) a $\pi$ CEP phase shift of the laser pulses during the streaking trace acquisition. Measurements of CEP fluctuations prove that it is not the latter. At this photon energy we expect multiple shells $[3 d(95 \mathrm{eV}), 4 s$
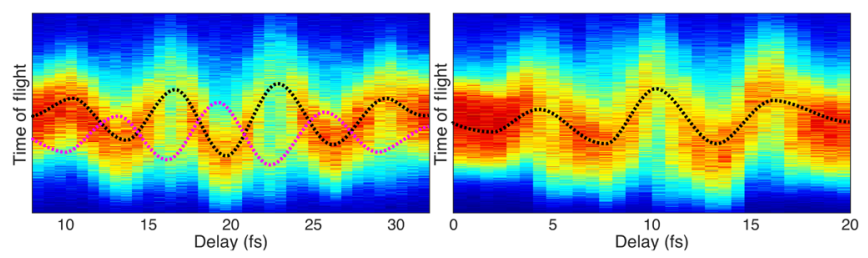

FIG. 5. Streaking traces performed using the same laser source, but a different HHG gas (argon), which offers higher flux at the expense of photon energy (centered $100 \mathrm{eV}$ ). The trace on the left for a randomly selected value of CEP clearly shows more than one attosecond pulse (highlighted with the dotted black and magenta curves). By selecting a value of CEP that results in a spectral continuum and maximum cutoff, the trace on the right is recorded, where only one attosecond pulse is detected. Argon gas is used primary for alignment, as the photon yield is higher and traces are acquired quicker; i.e., the full traces are acquired in 5 and $20 \mathrm{~min}$, respectively.

$(27.5 \mathrm{eV})$, and $4 p(14.1 \mathrm{eV})]$ to contribute due to their comparable ionization cross sections. If emission originated from multiple shells though, their corresponding emitted bandwidths would overlap; however, we (a) do not observe interference between them and (b) expect the delay between emission from different shells to be on the order of a couple of tens of attoseconds [4], so this could not explain what is seen in Fig. 5. Double pulse ghosting resulting from two attosecond bursts generated by subsequent half-cycles has been investigated theoretically (see Fig. 2 of Ref. [64] and Ref. [65]). This implies that if there are any satellite attosecond pulses, they are of significantly lower intensity and their contribution to the photoelectron spectra is negligible. In these conditions, a sufficiently isolated, water-window attosecond pulse has been generated and partially characterized.

\section{Auger processes in SXR streaking}

As described above, the energy range and bandwidth of our x-ray pulses can access core shells of krypton. From Fig. 1, we find that the cross section for photoionization from the $3 d$ shell at $300 \mathrm{eV}$ is roughly 1 order of magnitude larger than from the $3 p$ shell and 2 orders of magnitude larger than from the $4 p$ shell. The asymmetry parameter of the $3 d$ and $3 p$ shells cross and are close to 1 at $300-\mathrm{eV}$ photon energy. The asymmetry parameters diverge slightly for lower and higher photon energies within the bandwidth of the pulse. Because of this behavior and because of the vastly different probability for ionization, we can safely assume that photoemission originates predominantly from the $3 d$ shell and that the asymmetry of the emission presents no impediment to our measurement. Still, it is critical to rule out any other sources of photoelectrons from ionization phenomena. Figure 6 shows the ion TOF spectrum for krypton ionized by the soft-x-ray continuum. The higher-order ionization indicates the presence of Auger processes. To quantify the contributions to the electron 


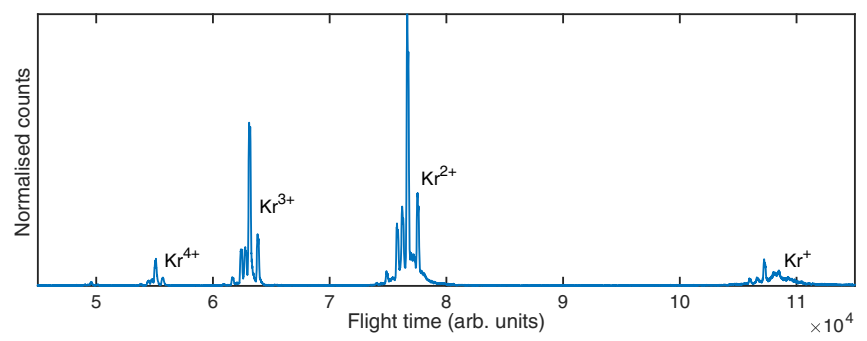

FIG. 6. Time-of-flight spectrum measured in krypton ionized by the soft-X-ray continuum. The presence of $\mathrm{Kr}^{2+}, \mathrm{Kr}^{3+}$, and $\mathrm{Kr}^{4+}$ clearly indicates the occurrence of Auger relaxation mechanism after the initial photoionization. The isotope structure of $\mathrm{Kr}$ is also visible.

spectra from the Auger processes that are expected from core-level ionization, we operate the time-of-flight spectrometer in an ion detection mode and record the ion spectra. Figure 6 shows the time-of-flight spectra illustrating clearly the second, third, and fourth ionization of the krypton gas, which is evidence of Auger relaxation processes. We perform a thorough analysis of the expected processes to ascertain whether our recorded streaking spectrogram contains contributions from them. Analysis of the partial ionization cross sections [66] indicates that ejection of a $3 d$ electron is the dominant ionization mechanism in krypton in the photon energy range between $94 \mathrm{eV}$ (threshold at $94.20 \mathrm{eV}$ ) and $300 \mathrm{eV}$. For very high photon energy, ionization from the $3 p$ shell is energetically allowed (threshold at $214.80 \mathrm{eV}$ [67]. In the energy range between 214 and $300 \mathrm{eV}$, the photoionization cross section from the $3 p$ shell is about $5 \%-10 \%$ [68] of the one from the $3 d$ shell, thus making this contribution negligible. The initial photoionization (from the $3 d$ and $3 p$ shell) step leads with high efficiency to the emission of one (or more) electron by Auger decay. This conclusion is confirmed by the ion spectra, which present multiple charged ion states $\mathrm{Kr}^{2+}, \mathrm{Kr}^{3+}$, and $\mathrm{Kr}^{4+}$ (see Fig. 6). For the interpretation of the streaking data, it is fundamental to understand in which energy range the Auger lines will appear and the different branching ratio between the different channels. For the sake of clarity, we discuss the two cases separately.

(i) Auger decay from the $3 d-1$ shell.-Ionization from the $3 d$ shell leads to single or cascaded Auger decay determining the formation of $\mathrm{Kr}^{2+}$ and $\mathrm{Kr}^{3+}$ ions, respectively. For both mechanisms, the energy of the Auger electrons is always below $60 \mathrm{eV}$ (see also Fig. 3 in Ref. [69]) and it does not affect the streaking trace, considering that only photoelectrons with energies larger that $90 \mathrm{eV}$ are efficiently collected.

(ii) Auger decay from the $3 p$ shell.-Experimental data on different Auger relaxation pathways of the core-hole $3 p$ are reported in Ref. [70]. In particular, three groups of Auger lines were analyzed and their main characteristics are summarized in Table II. These Auger decays fall out of the main energetic window of the TOF and are not
TABLE II. Analysis of the Auger decay following photoionization from the $3 p$ core shell.

\begin{tabular}{lcc}
\hline \hline Auger process & Threshold (eV) & Branching ratio \\
\hline $\mathrm{M}_{2,3}-\mathrm{M}_{4,5} \mathrm{~N}_{1}$ & $57-90$ & $21.3 \%$ \\
$\mathrm{M}_{2,3}-\mathrm{M}_{4,5} \mathrm{~N}_{2,3}$ & $85-105$ & $75.4 \%$ \\
$\mathrm{M}_{2,3}-\mathrm{NN}$ & $150-185$ & $2.3 \%$ \\
\hline \hline
\end{tabular}

efficiently collected by our electron spectrometer. The relative intensity of $\mathrm{M}_{2,3}-\mathrm{M}_{4,5} \mathrm{~N}_{2,3}$ group is, according to Ref. [70], about $75.4 \%$. According to Ref. [71], the relative intensity of these decay channels is given by $\mathrm{M}_{2}-\mathrm{M}_{4,5} \mathrm{~N}_{2,3}, 50.6 \%$, and $\mathrm{M}_{3}-\mathrm{M}_{4,5} \mathrm{~N}_{2,3}, 61.4 \%$. Therefore, the Auger lines are only up to $6 \%$ of the total $3 d$ signal and can be safely neglected. Finally, the ratio of the $\mathrm{M}_{2,3}$-NN Auger lines with respect to the direct photoionization from the $3 d$ shell is given by $\mathrm{M}_{2,3}-\mathrm{NN} / 3 d=$ $0.66 \%$ and can be neglected. The main conclusion of our analysis is that the Auger decay processes do not appreciably contribute to the photoelectron spectrum measured in our experimental conditions; the electrons measured are emitted by single-photon ionization from the $3 d$ shell.

It is important to note that any attosecond photoelectron-based streaking method retrieves the electron wave packet and not the optical pulse directly and irrespective of the used retrieval method. The typical assumption is therefore the identical mapping of the optical pulse into the measured electron wave packet. Because of the large bandwidth of the electron wave packet, we investigate whether the typical assumption holds or if any significant time lag is expected for photoemission from the $3 d$ shell of krypton. Figure 7 shows the calculated dipole emission phase as a function of photon energy [72]. The $3 d$ phase is mostly featureless across the relevant photon energy range for which we extract a GDD of $-10.7 \mathrm{as}^{2}$ at $243 \mathrm{eV}$. This negligible contribution of the dipole emission phase means that the retrieved electron wave packet is indeed an accurate representation of the soft-x-ray attosecond pulse.

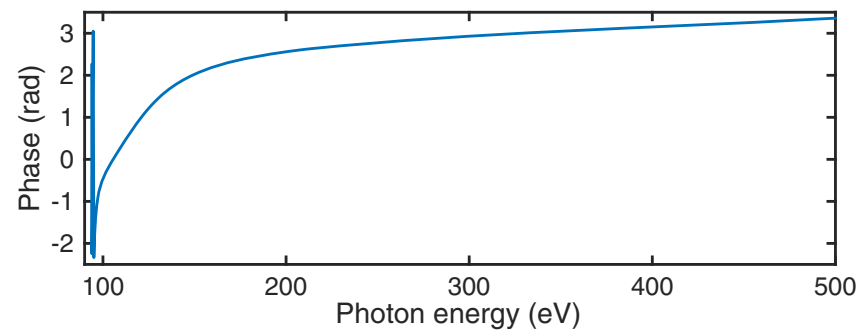

FIG. 7. Calculated dipole emission phase for $\mathrm{Kr} 3 d$ as a function of photon energy [72]. 


\section{Retrieval of attosecond SXR pulses}

In the following, we use the FROGCRAB [47] algorithm to retrieve information about the water-window pulse from our measured streaking spectrogram. We note that while different algorithms are nowadays available to improve on various aspects of FROGCRAB, we decided to use FROGCRAB as it is the most widely applied method in the attosecond field. An important approximation made in many algorithms, and also in FROGCRAB, is the central momentum approximation (CMA), which requires that the pulse's spectral bandwidth is not larger than the central photon energy of the spectrum [73]. While there exists no hard boundary for the validity of the CMA, we note that the extreme bandwidth of our pulse approaches this limit but does not clearly violate it. We like to stress, however, that similarly to FROG retrievals of optical pulses, an extreme pulse bandwidth and any measurement with low signal-tonoise ratio, as currently only possible in the SXR regime, places stringent demands on the sampling conditions and convergence criteria.

It is important to note that interpolation and filtering of spectrogram traces is a common practice, but each case should be carefully considered before applying such measures. In our example, optimal spectral sampling $\delta E$, according to Table III, would require measuring with an order of magnitude better TOF resolution, but the corresponding retrieval grid would become impractically large to handle numerically. Without such fine sampling, one may, however, neglect to reveal spectral interferences originating from multiple pulses or substructures of the attosecond pulse. To rule out such possibilities, we take additional photon spectra with a resolution of $0.5 \mathrm{eV}$. These spectra do not reveal any fringes or fine structures, hence supporting the measurement of an isolated water-window SXR pulse. In addition, we simulate a single-cycle streaking spectrogram with $\delta E$ of $0.2 \mathrm{eV}$, which is then interpolated to $\delta E$ of $0.6 \mathrm{eV}$. We choose $0.6 \mathrm{eV}$ instead of the optimally required $0.3 \mathrm{eV}$ since reconstruction time is still tractable but already amounts to $48 \mathrm{~h}$. The interpolated trace is processed by the FROGCRAB algorithm and satisfactorily reconstructs the simulated pulse. Moreover, we verify numerically that the interpolation factor we use still results in a reliable FROGCRAB reconstruction: The simulated trace is then downsized to have $\delta E$ of $10 \mathrm{eV}$ to emulate a low-resolution spectrometer and afterwards reinterpolated to a $\delta E$ of $0.6 \mathrm{eV}$. This new heavily

TABLE III. FROGCRAB parameters.

\begin{tabular}{ll}
\hline \hline Parameter & Value \\
\hline$\delta E$ & $0.34 \mathrm{eV}$ \\
$\delta \tau$ & $5.9 \mathrm{as}$ \\
$N$ & 2048 \\
$\Delta \tau$ (total time delay range) & $12 \mathrm{fs}$ \\
$\Delta E$ (total energy range) & $700 \mathrm{eV}$ \\
\hline \hline
\end{tabular}

interpolated trace is then processed by the FROGCRAB algorithm and similarly reconstructs and supports a single pulse. In general, such a procedure should be considered on a case-by-case basis since it is a common mistake to use insufficient sampling points and strong filtering, thereby neglecting the fine details within a spectrogram. Quoting marginals and FROG errors are also only meaningful if the measurement grid is sufficiently populated with data above the noise level. Whatever the conditions may be, temporal structures should be well resolved and reflected also in the spectral domain, and visa versa.

The principal component generalized projections algorithm (PCGPA) iterative loop used by the FROGCRAB algorithm relies on several numerical constraints for the data set to be processed [65]. One of these requires the data matrix to be squared, with the number of points on each axis $N$ being a power of 2 and satisfying the sampling criterion, which connects the resolution of the frequency and time-delay axis with the relation

$$
\delta \tau \delta E=\frac{2 \pi \hbar}{e N},
$$

where $E$ is the energy expressed in $\mathrm{eV}$ and $e$ is the unit electron charge. For our experimental trace, bandwidth exceeds an energy range of $200 \mathrm{eV}$, setting a lower limit for the total energy (frequency) range where the data need to be interpolated. For the delay axis, without knowing the pulse duration, we set the time-delay resolution to be smaller than the Fourier transform limit (20 as) so as to have sufficient points for the reconstruction. Finally, the accuracy of the reconstruction increases with the number of points $N$, but so obviously does the computational time needed by the computer to run the algorithm. Taking into account all these features, we finally choose the parameters listed in Table III to perform the FROGCRAB retrievals.

Based on these parameters, we apply the FROGCRAB algorithm separately to each of the two cycles shown in the streaking trace in Fig. 4. Within the error, both of the retrievals should give the same information about the SXR pulse. Figure 8 shows the results in which we show the experimental trace for each cycle (top and bottom row) next to the reconstructed trace and the temporal profile with instantaneous frequency. We extract pulse durations of 23.1 and 24.1 as, and lowest-order phase of 152 and 274 as $^{2}$, respectively.

\section{E. Contributions to chirp compensation}

As a next step we try to investigate possible sources that would lead to chirp compensation and the generation of a SXR pulse below the atomic unit of time. In contrast to the $\mathrm{XUV}$, this is a difficult undertaking in the SXR regime, as the ubiquitous reference measurement with purposely added chirp by means of a metal filter is not applicable anymore in the high-photon energy range above $250 \mathrm{eV}$. Moreover, relying on simulations is also very limited, as we 

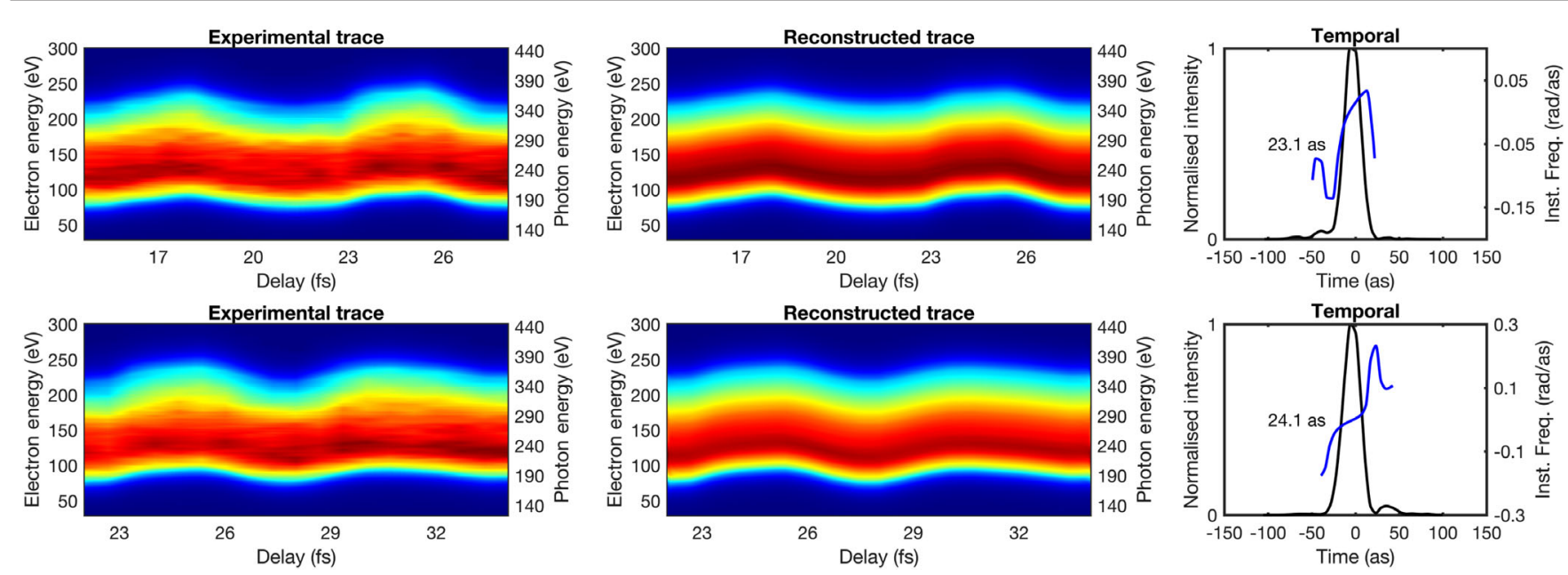

FIG. 8. FROGCRAB retrievals of the raw data shown in Fig. 4. Top: Two cycles from 15-27 fs; bottom: two cycles from $22-34$ fs. Lefthand plots show the measured traces which have had some filtering applied. Middle plots show the FROGCRAB reconstructed traces and the right-hand plots show their temporal profiles.

are not aware of any simulation that can describe HHG under such high pressures in combination with full 3D propagation. As a first crude measure, we hence calculate the classical attochirp as expected for a single emitter to $2550 \mathrm{as}^{2}$. Investigating any possible contribution to phase compensation, we first turn to the remnant gas flowing from the high-pressure gas target. Simulations based on computational fluid dynamics of the gas pressure on axis, reveal a value of $-165 \mathrm{as}^{2}$. Moreover, we find that dispersion contributions from the plasma are negligible at these photon energies. Lastly, the dipole phase associated with the ionization of the krypton streaking target gas is both relatively flat as well as negligible over our bandwidth.

\section{F. Temporal gating at SXR generation conditions}

Despite the absence of any obvious postgeneration chirp compensation, we mention that recent research has presented evidence that the high-pressure conditions for SXR pulse generation provide the possibility for near-instantaneous temporal gating which could lead to the emission of extremely short, isolated SXR pulses. First investigations [42] showed a transition from attosecond pulse train generation to isolated attosecond pulse generation despite using a multicycle pulse when increasing pressure. Our own investigation [74], for the much higher pressures (10 bar) used in our case in He, suggested that through the interplay of gas pressure, intensity, and wavelength, a short temporal phase-matching window exists. In the regime of long wavelength, high-intensity laser pulses, and a high gas pressure target, the temporal phase-matching window can be much less than half a cycle of the laser pulse. Here, we perform a similar simulation of the exact experimental conditions. Our phase-matching calculations consider dispersion of both wavelengths from neutral gas and free electrons, the geometric phase (Gouy phase) of the fundamental as well as the dipole phase of the short trajectories. We do not consider long trajectories since they are effectively suppressed during propagation due to their larger divergence emission, filtered by the various pumping apertures of our beam line. Absorption of the propagating radiation is also considered. For the radiation generation, a semiclassical model of HHG is considered with the strong field approximation. A Ne atom is tunnel ionized by a linearly polarized electric field with a peak intensity of $4.3 \times 10^{14} \mathrm{~W} / \mathrm{cm}^{2}$, a central wavelength of $1850 \mathrm{~nm}$, and a pulse duration of $12 \mathrm{fs}$ FWHM with a Gaussian temporal profile and a Gaussian focus with a waist of $54 \mu \mathrm{m}$. These parameters are used to find the timedependent fraction of free electrons. High harmonics are generated from a single electron from the outer shell of each atom. The calculations then consider the ion in the ground state. The ionization rates are calculated using the Ammosov, Delone, Krainov (ADK) formula [75]. We use a continuous wave approximation where the electric field amplitude varies very slowing during the tunneling process and the dipole approximation where the driving laser field wavelength is significantly larger than the electron wave function. The tunnel-ionized electron is considered not to interact with the remaining ion and to have zero initial kinetic energy. It is assumed that the ion remaining after ionization does not interact with the laser field. The effect of the magnetic field of the driving laser is neglected. The results are show in Fig. 9, illustrating that for our experimental conditions, the phase mismatch $(\Delta k)$ approaches zero, supporting the conditions for an extremely short isolated attosecond burst.

While these simulations, and the previous publications, are not conclusive to place a value on the expected duration of the attosecond pulse, they clearly point to the different generation conditions in the SXR regime and our measurement is the first attosecond streaking-based experimental verification of SXR gating via temporal phase matching, 


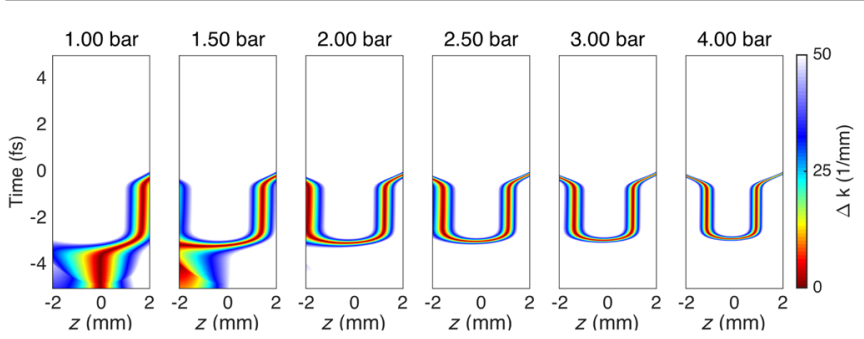

FIG. 9. Spatiotemporal phase-matching maps as a function of HHG target pressure in Ne. Calculated on-axis phase mismatch as a function of propagation position and time within the pulse for $300-\mathrm{eV}$ radiation generated in neon for our experimental conditions and target pressures of 1-4 bar. The intensity in our target only has a field strength sufficient to generate $300 \mathrm{eV}$ radiation between -1 to $1 \mathrm{~mm}$ in the propagation direction.

resulting in an isolated water-window attosecond pulse. Additional evidence for the emission of an isolated attosecond burst comes from the spectral continuum generated during HHG. The spectrometer has a resolution below $0.5 \mathrm{eV}$, which is less than the spacing between discrete harmonics driven by 1850 -nm radiation $(1.4 \mathrm{eV})$. The lack of spectral modulations or discrete harmonics suggests an isolated attosecond pulse is generated. Without a direct possibility to infer the duration of the isolated attosecond pulse, we can, however, place an upper limit of 322 as on the pulse duration based on the classically estimated attochirp of $2385 \mathrm{as}^{2}$ (intrinsic attochirp, minus the remnant gas dispersion) and the pulse bandwidth.

\section{G. Requirements for streaking at high-photon energies}

Without limitation to the possibility of intrinsic temporal gating, we investigate additional reasons why no asymmetry in the streaking trace could be visible. Clearly, the ultrabroad bandwidth responsible for these attosecond pulses pushes the streaking technique and the FROGCRAB algorithm into a new and extreme regime. Here, we
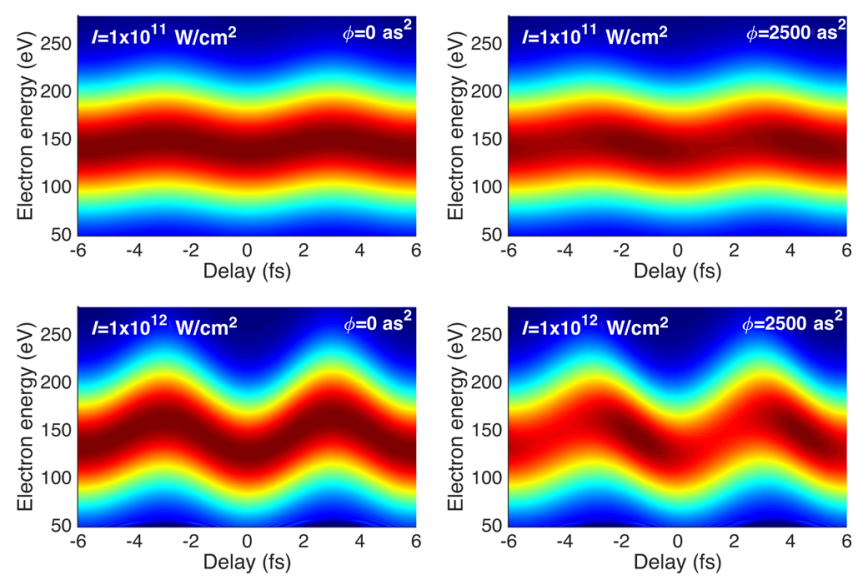

FIG. 10. Noise-free streaking field intensity simulations. Top: Low intensity; bottom: high intensity. Left: No attochirp simulated; right: $2500 \mathrm{as}^{2}$ simulated. investigate theoretically the influence of two important and interlinked parameters: bandwidth and streaking field intensity. The streaking excursion is proportional to the field strength of the streaking pulse; we use a $1850-\mathrm{nm}$ pulse at a streaking field intensity of $10^{11} \mathrm{~W} / \mathrm{cm}$.

We first examine the influence of the streaking field intensity on a streaking spectrogram having a fixed and broad bandwidth similar to our experimentally measured bandwidth. Figure 10 shows theoretically generated, noisefree streaking spectrograms composed of two cycles for our bandwidth. The left plots have no chirp applied and the right plots have $2500 \mathrm{as}^{2}$ applied. The results show that for an intensity of $10^{11} \mathrm{~W} / \mathrm{cm}^{2}$ the streaking asymmetry (leading versus trailing edge) is barely visible, whereas for $10^{12} \mathrm{~W} / \mathrm{cm}^{2}$ it is clearly visible. Without adding experimental noise, this may suggest that a lack of asymmetry arises from insufficient streaking intensity.
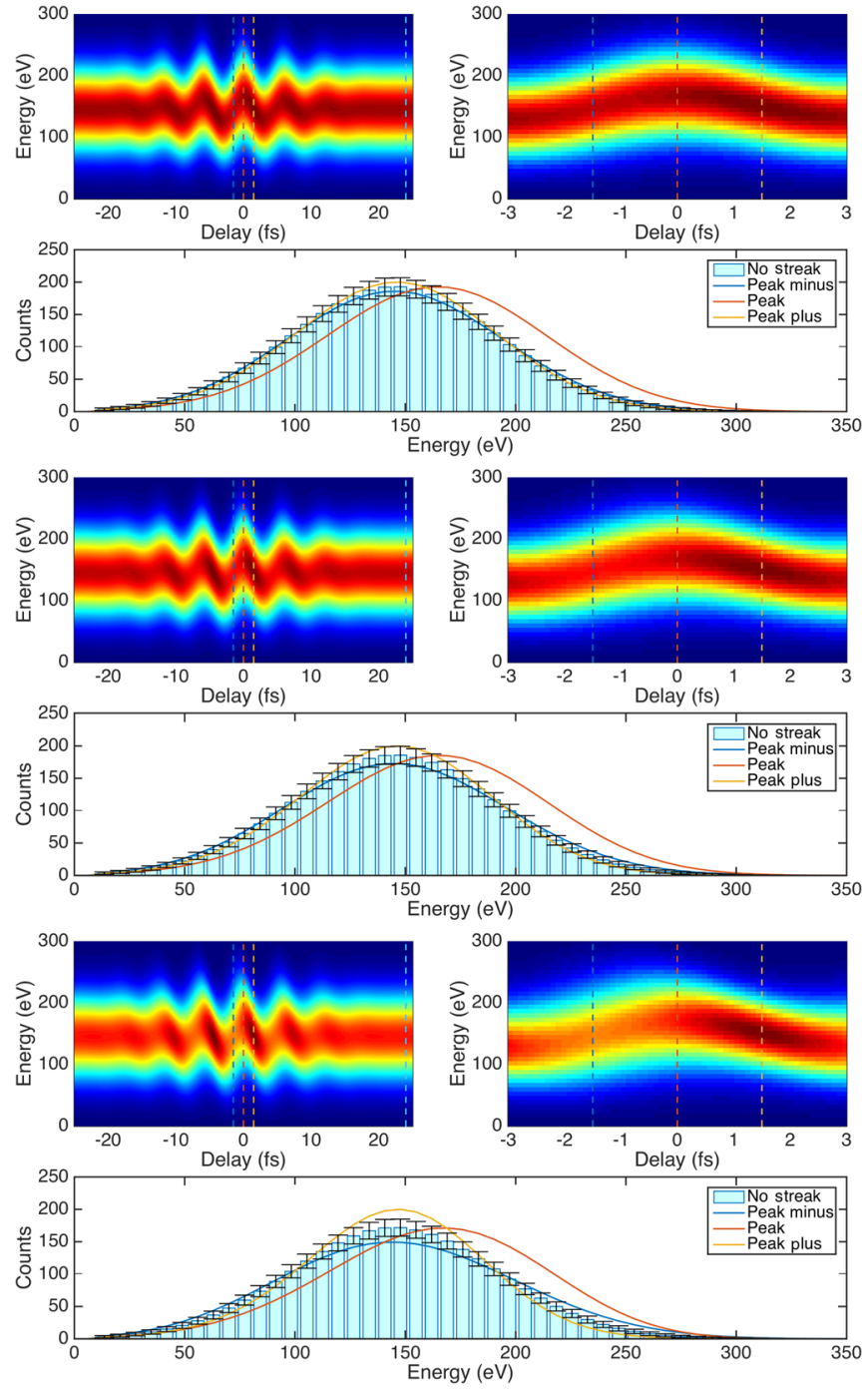

FIG. 11. Statistical noise analysis of an attosecond pulse with similar bandwidth to our experimental data. Top: $1250 \mathrm{as}^{2}$ simulated; middle: $2500 \mathrm{as}^{2}$ simulated; bottom: $5000 \mathrm{as}^{2}$ simulated. 


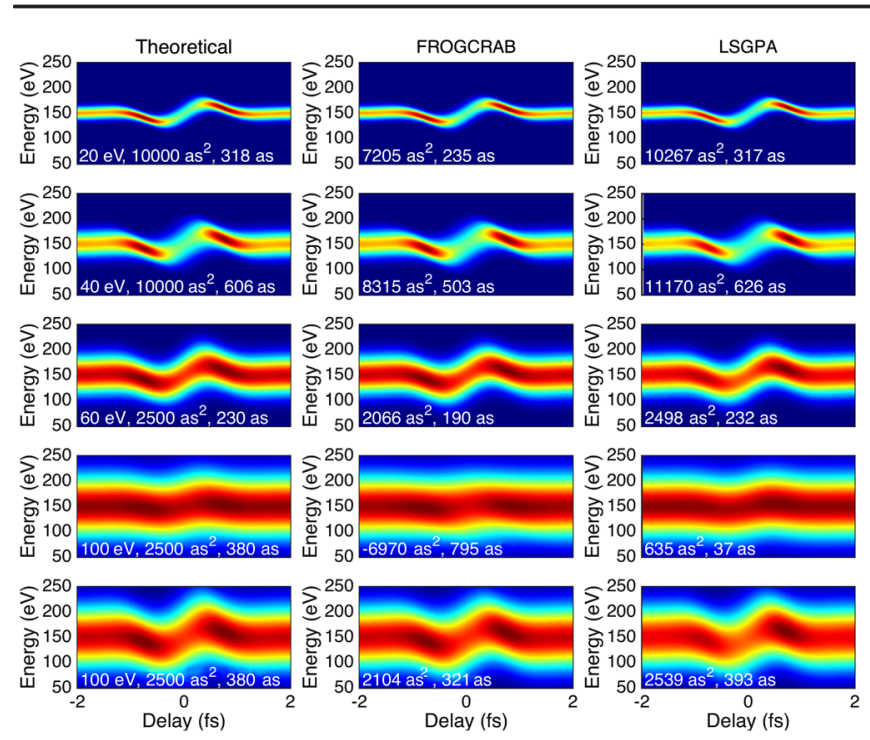

FIG. 12. Bandwidth retrievals. Left-hand column (theoretically generated streaking spectrograms): Plots are annotated at the bottom left, with the simulated bandwidth, simulated applied attochirp, and corresponding simulated pulse duration. Middle (FROGCRAB algorithm) and right-hand (LSGPA algorithm) columns are annotated with the retrieved attochirp and the retrieved pulse duration. Top four rows: $10^{11} \mathrm{~W} / \mathrm{cm}^{2}$. Bottom row: $10^{12} \mathrm{~W} / \mathrm{cm}^{2}$.

However, a detrimental requirement is to avoid direct ionization from the streaking field, which places an upper limit to the intensity. To investigate this dependency further, we simulate the influence of noise on generated streaking traces based on our experimental bandwidth and chirp parameters. Three simulation sets shown in Fig. 11 are generated having 1250,2500 , and $5000 \mathrm{as}^{2}$ chirp, respectively. A lineout of the photoelectron spectrum is taken for an unstreaked value. Error bars are assigned to the lineout based on Poissonian statistics. Lineouts taken at the maximum and zero crossings are then compared to the error bars. For a statistically relevant asymmetry at the zero crossings, the yellow and blue curves need to equal or exceed the error bars. The results suggest that for a low signal-to-noise ratio, even when asymmetry is fairly obvious, an attochirp of $2500 \mathrm{as}^{2}$ is barely statistically discernible, whereas at $5000 \mathrm{as}^{2}$ it is.

To gain further understanding of the limits due to the CMA, we investigate the capability of the FROGCRAB algorithm as well as the least-squares generalized projections algorithm (LSGPA) [65] to retrieve the pulse duration as a function of increasing bandwidth. The results are shown in Fig. 12 in which bandwidth is increased from 20 to $100 \mathrm{eV}$. For bandwidths up to $60 \mathrm{eV}$, both algorithms satisfactorily reconstruct the theoretical pulses even with a moderate streaking field intensity of $10^{11} \mathrm{~W} / \mathrm{cm}^{2}$. For a $100-\mathrm{eV}$ bandwidth and streaking field intensity of $10^{11} \mathrm{~W} / \mathrm{cm}^{2}$, both reconstruction algorithms fail. This highlights the need for a sufficiently intense streaking pulse to facilitate successful reconstruction of the attosecond pulse. This is illustrated by the bottom set of plots in which a broad spectrum similar to our measured spectrum, combined with sufficient streaking field intensity $\left(10^{12} \mathrm{~W} / \mathrm{cm}^{2}\right)$, facilitates the successful reconstruction with both the FROGCRAB and LSGPA algorithm.

We note that although we focus on the most well-known retrieval algorithm (FROGCRAB), it is important to stress that the conclusions we draw in this work pertain to the physics of the experimental data acquisition. Independent of the retrieval algorithm chosen to process the data, in this regime of extremely broad spectra and high-photon energy, diligence and attention to detail are required in the choice of photoelectron source gas, streaking field intensity, and signal-tonoise ratio. Ignoring any of these factors could lead to misleading pulse retrievals from all of the available algorithms.

\section{SUMMARY}

We perform the first streaking measurement of waterwindow photon-energy pulses. The pulses are generated via $\mathrm{HHG}$ in $\mathrm{Ne}$ and are driven by sub-2-cycle CEP-stable laser pulses with a central wavelength of $1850 \mathrm{~nm}$. The streaking traces acquired do not exhibit any obvious sign of attochirp, which we may attribute to either issues with the measurement process or a newly found generating condition at the unprecedentedly high pressures and ionization conditions [74]. Notwithstanding these limitations, we are able to confirm the generation of an isolated attosecond water-window SXR pulse and we can place an upper bound on the pulse duration of 322 as. Simulations indicate that in this ultrabroadband regime, the streaking excursion needs to be similar in magnitude to the bandwidth and that good signal-to-noise ratio has to be experimentally achieved. Further simulations indicate that when attosecond streaking is combined with either the FROGCRAB or the LSGPA algorithms, reconstruction in an ultrabroadband regime is possible as long as the streaking excursion and signal-to-noise ratio requirements are met. Our next step will be to increase the streaking field intensity and improve the signal-to-noise ratio in order to fully characterize the water-window pulses that we are generating.

\section{ACKNOWLEDGMENTS}

The authors acknowledge the Spanish Ministry of Economy and Competitiveness "Severo Ochoa" Programme for Centres of Excellence in R\&D (SEV2015-0522), FIS2014-56774-R, Catalan Agencia de Gestió d'Ajuts Universitaris i de Recerca (AGAUR) SGR 2014-2016, Fundació Cellex Barcelona, CERCA Programme/Generalitat de Catalunya, Laserlab-Europe (EU-H2020 654148). We acknowledge fruitful discussions with Dr. Stefan Pabst, Dr. Jan Marcus Dahlström, Professor Chii-Dong Lin, Professor Zenghu Chang, and Professor Michael Chini. 
[1] P. B. Corkum and F. Krausz, Attosecond Science, Nat. Phys. 3, 381 (2007).

[2] M. Drescher, M. Hentschel, R. Kienberger, M. Uiberacker, V. Yakovlev, A. Scrinzi, Th. Westerwalbesloh, U. Kleineberg, U. Heinzmann, and F. Krausz, Time-Resolved Atomic Inner-Shell Spectroscopy, Nature (London) 419, 803 (2002).

[3] M. Uiberacker, Th. Uphues, M. Schultze, A. J. Verhoef, V. Yakovlev, M. F. Kling, J. Rauschenberger, N. M. Kabachnik, H. Schröder, M. Lezius, K. L. Kompa, H.-G. Muller, M. J. J. Vrakking, S. Hendel, U. Kleineberg, U. Heinzmann, M. Drescher, and F. Krausz, Attosecond RealTime Observation of Electron Tunnelling in Atoms, Nature (London) 446, 627 (2007).

[4] M. Schultze et al., Delay in Photoemission, Science 328, 1658 (2010).

[5] K. Klünder, J. M. Dahlström, M. Gisselbrecht, T. Fordell, M. Swoboda, D. Guénot, P. Johnsson, J. Caillat, J. Mauritsson, A. Maquet, R. Taïeb, and A. L'Huillier, Probing Single-Photon Ionization on the Attosecond Time Scale, Phys. Rev. Lett. 106, 143002 (2011).

[6] C. Ott, A. Kaldun, P. Raith, K. Meyer, M. Laux, J. Evers, C. H. Keitel, C. H. Greene, and T. Pfeifer, Lorentz Meets Fano in Spectral Line Shapes: A Universal Phase and Its Laser Control, Science 340, 716 LP-720 (2013).

[7] G. Sansone, F. Kelkensberg, J. F. Perez-Torres, F. Morales, M. F. Kling, W. Siu, O. Ghafur, P. Johnsson, M. Swoboda, E. Benedetti, F. Ferrari, F. Lepine, J. L. Sanz-Vicario, S. Zherebtsov, I. Znakovskaya, A. L'Huillier, M. Yu. Ivanov, M. Nisoli, F. Martin, and M. J.J. Vrakking, Electron Localization Following Attosecond Molecular Photoionization, Nature (London) 465, 763 (2010).

[8] F. Calegari, D. Ayuso, A. Trabattoni, L. Belshaw, S. De Camillis, S. Anumula, F. Frassetto, L. Poletto, A. Palacios, P. Decleva, J. B. Greenwood, F. Martín, and M. Nisoli, Ultrafast Electron Dynamics in Phenylalanine Initiated by Attosecond Pulses., Science 346, 336 (2014).

[9] A. Schiffrin, T. Paasch-Colberg, N. Karpowicz, V. Apalkov, D. Gerster, S. Muhlbrandt, M. Korbman, J. Reichert, M. Schultze, S. Holzner, J. V. Barth, R. Kienberger, R. Ernstorfer, V. S. Yakovlev, M. I. Stockman, and F. Krausz, Optical-Field-Induced Current in Dielectrics, Nature (London) 493, 70 (2013).

[10] M. Schultze, K. Ramasesha, C. D. Pemmaraju, S. A. Sato, D. Whitmore, A. Gandman, J. S. Prell, L. J. Borja, D. Prendergast, K. Yabana, D. M. Neumark, and S. R. Leone, Attosecond Band-Gap Dynamics in Silicon, Science 346, 1348 (2014).

[11] M. Hentschel, R. Kienberger, C. Spielmann, G. A. Reider, N. Milosevic, T. Brabec, P. B. Corkum, U. Heinzmann, M. Drescher, and F. Krausz, Attosecond Metrology, Nature (London) 414, 509 (2001).

[12] G. Sansone, E. Benedetti, F. Calegari, C. Vozzi, L. Avaldi, R. Flammini, L. Poletto, P. Villoresi, C. Altucci, R. Velotta, S. Stagira, S. De Silvestri, and M. Nisoli, Isolated Single-Cycle Attosecond Pulses, Science 314, 443 (2006).

[13] E. Goulielmakis, M. Schultze, M. Hofstetter, V. S. Yakovlev, J. Gagnon, M. Uiberacker, A. L. Aquila, E. M. Gullikson, D. T. Attwood, R. Kienberger, F. Krausz, and
U. Kleineberg, Single-Cycle Nonlinear Optics, Science 320, 1614 (2008).

[14] F. Ferrari, F. Calegari, M. Lucchini, C. Vozzi, S. Stagira, G. Sansone, and M. Nisoli, High-Energy Isolated Attosecond Pulses Generated by Above-Saturation Few-Cycle Fields, Nat. Photonics 4, 875 (2010).

[15] K. Zhao, Q. Zhang, M. Chini, Y. Wu, X. Wang, and Z. Chang, Tailoring a 67 Attosecond Pulse through Advantageous Phase-Mismatch, Opt. Lett. 37, 3891 (2012).

[16] M. Fieß, M. Schultze, E. Goulielmakis, B. Dennhardt, J. Gagnon, M. Hofstetter, R. Kienberger, and F. Krausz, Versatile Apparatus for Attosecond Metrology and Spectroscopy, Rev. Sci. Instrum. 81, 093103 (2010).

[17] S. Svensson, B. Eriksson, N. Mårtensson, G. Wendin, and U. Gelius, Electron Shake-Up and Correlation Satellites and Continuum Shake-Off Distributions in X-Ray Photoelectron Spectra of the Rare Gas Atoms, J. Electron Spectrosc. Relat. Phenom. 47, 327 (1988).

[18] N. Sisourat, N. V. Kryzhevoi, P. Kolorenc, S. Scheit, T. Jahnke, and L.S. Cederbaum, Ultralong-Range Energy Transfer by Interatomic Coulombic Decay in an Extreme Quantum System, Nat. Phys. 6, 508 (2010).

[19] T.-C. Tseng, C. Urban, Y. Wang, R. Otero, S. L. Tait, M. Alcamí, D. Écija, M. Trelka, J. M. Gallego, N. Lin, M. Konuma, U. Starke, A. Nefedov, A. Langner, C. Wöll, M. Á. Herranz, F. Martín, N. Martín, K. Kern, and R. Miranda, Charge-Transfer-Induced Structural Rearrangements at Both Sides of Organic/Metal Interfaces, Nat. Chem. 2, 374 (2010).

[20] F. Remacle and R. D. Levine, An Electronic Time Scale in Chemistry, Proc. Natl. Acad. Sci. U.S.A. 103, 6793 (2006).

[21] G. Griffini, L. Brambilla, M. Levi, M. D. Zoppo, and S. Turri, Photo-Degradation of a Perylene-Based Organic Luminescent Solar Concentrator: Molecular Aspects and Device Implications, Sol. Energy Mater. Sol. Cells 111, 41 (2013).

[22] M. Ross, Matter under Extreme Conditions of Temperature and Pressure, Rep. Prog. Phys. 48, 1 (1985).

[23] J. Stähler, U. Bovensiepen, and M. Wolf, Dynamics at Solid State Surfaces and Interfaces: Current Developments (Wiley, New York, 2010), Vol. 1, pp. 359-379.

[24] M. Shanmugam, R. Jacobs-Gedrim, C. Durcan, and B. Yu, $2 D$ Layered Insulator Hexagonal Boron Nitride Enabled Surface Passivation in Dye Sensitized Solar Cells, Nanoscale 5, 11275 (2013).

[25] F. Benjamin, F. Zamponi, V. Juvé, J. Stingl, M. Woerner, T. Elsaesser, and M. Chergui, Ultrafast Inter-Ionic Charge Transfer of Transition-Metal Complexes Mapped by Femtosecond X-Ray Powder Diffraction, J. Chem. Phys. 138, 144504 (2013).

[26] A. Fohlisch, P. Feulner, F. Hennies, A. Fink, D. Menzel, D. Sanchez-Portal, P. M. Echenique, and W. Wurth, Direct Observation of Electron Dynamics in the Attosecond Domain, Nature (London) 436, 373 (2005).

[27] R. Boll et al., Femtosecond Photoelectron Diffraction on Laser-Aligned Molecules: Towards Time-Resolved Imaging of Molecular Structure, Phys. Rev. A 88, 061402 (2013).

[28] J. Breidbach and L.S. Cederbaum, Universal Attosecond Response to the Removal of an Electron, Phys. Rev. Lett. 94, 033901 (2005). 
[29] J. P. Reed, B. Uchoa, Y. I. Joe, Y. Gan, D. Casa, E. Fradkin, and P. Abbamonte, The Effective Fine-Structure Constant of Freestanding Graphene Measured in Graphite, Science 330, 805 (2010).

[30] S. L. Cousin, F. Silva, S. Teichmann, M. Hemmer, B. Buades, and J. Biegert, High-Flux Table-Top Soft X-Ray Source Driven by Sub-2-Cycle, CEP Stable, 185- $\mu \mathrm{m} 1-\mathrm{kHz}$ Pulses for Carbon K-Edge Spectroscopy, Opt. Lett. 39, 5383 (2014).

[31] S. L. Cousin, F. Silva, S. Teichmann, M. Hemmer, B. Buades, and J. Biegert, in Proceedings of the Conference on Ultrafast Optics (UFO X), Beijing, 2015 (2015), http:// www.ultrafastoptics2015.org/EN/column/column1.shtml.

[32] J. Biegert, in Proceedings of the 47th Annual Meeting of the APS Division of Atomic, Molecular and Optical Physics (American Physical Society, New York, 2016) p. BAPS.2016.DAMOP.U9.2.

[33] D. J. Kane and R. Trebino, Characterization of Arbitrary Femtosecond Pulses Using Frequency-Resolved Optical Gating, IEEE J. Quantum Electron. 29, 571 (1993).

[34] I. A. Walmsley and V. Wong, Characterization of the Electric Field of Ultrashort Optical Pulses, J. Opt. Soc. Am. B 13, 2453 (1996).

[35] M. Nisoli, S. De Silvestri, and O. Svelto, Generation of High Energy $10 \mathrm{fs}$ Pulses by a New Pulse Compression Technique, Appl. Phys. Lett. 68, 2793 (1996).

[36] B. Schenkel, J. Biegert, U. Keller, C. Vozzi, M. Nisoli, G. Sansone, S. Stagira, S. De Silvestri, and O. Svelto, Generation of 3.8-fs Pulses from Adaptive Compression of a Cascaded Hollow Fiber Supercontinuum, Opt. Lett. 28, 1987 (2003).

[37] C. P. Hauri, W. Kornelis, F. W. Helbing, A. Heinrich, A. Couairon, A. Mysyrowicz, J. Biegert, and U. Keller, Generation of Intense, Carrier-Envelope Phase-Locked Few-Cycle Laser Pulses through Filamentation, Appl. Phys. B 79, 673 (2004).

[38] A. Couairon, M. Franco, A. Mysyrowicz, J. Biegert, and U. Keller, Pulse Self-Compression to the Single-Cycle Limit by Filamentation in a Gas with a Pressure Gradient, Opt. Lett. 30, 2657 (2005).

[39] C. Hauri, A. Guandalini, P. Eckle, W. Kornelis, J. Biegert, and U. Keller, Generation of Intense Few-Cycle Laser Pulses through Filamentation-Parameter Dependence, Opt. Express 13, 7541 (2005).

[40] A. Guandalini, P. Eckle, M. Anscombe, P. Schlup, J. Biegert, and U. Keller, 5.1 fs Pulses Generated By Filamentation and Carrier Envelope Phase Stability Analysis, J. Phys. B 39, S257 (2006).

[41] A. Wirth, M. Th. Hassan, I. Grguraś, J. Gagnon, A. Moulet, T. T. Luu, S. Pabst, R. Santra, Z. A. Alahmed, A. M. Azzeer, V. S. Yakovlev, V. Pervak, F. Krausz, and E. Goulielmakis, Synthesized Light Transients, Science 334, 195 (2011).

[42] M. C. Chen, C. Mancuso, C. Hernández-García, F. Dollar, B. Galloway, D. Popmintchev, P.-C. Huang, B. Walker, L. Plaja, A. Jaroń-Becker, A. Becker, M. M. Murnane, H. C. Kapteyn, and T. Popmintchev, Generation of Bright Isolated Attosecond Soft X-Ray Pulses Driven by Multicycle Midinfrared Lasers, Proc. Natl. Acad. Sci. U.S.A. 111, 1 (2014).
[43] P. B. Corkum, Plasma Perspective on Strong-Field Multiphoton Ionization, Phys. Rev. Lett. 71, 1994 (1993).

[44] K. T. Kim, C. Zhang, T. Ruchon, and J. F. Hergott, Photonic Streaking of Attosecond Pulse Trains, Nat. Photonics 7, 651 (2013).

[45] J. Itatani, F. Quéré, G. L. Yudin, M. Yu Ivanov, F. Krausz, and P. B. Corkum, Attosecond Streak Camera, Phys. Rev. Lett. 88, 173903 (2002).

[46] P. M. Paul, E. S. Toma, P. Breger, G. Mullot, F. Augé, Ph. Balcou, H. G. Muller, and P. Agostini, Observation of a Train of Attosecond Pulses from High Harmonic Generation, Science 292, 1689 (2001).

[47] Y. Mairesse and F. Quéré, Frequency-Resolved Optical Gating for Complete Reconstruction of Attosecond Bursts, Phys. Rev. A 71, 011401 (2005).

[48] R. Kienberger, E. Goulielmakis, M. Uiberacker, A. Baltuska, V. Yakovlev, F. Bammer, A. Scrinzi, Th. Westerwalbesloh, U. Kleineberg, U. Heinzmann, M. Drescher, and F. Krausz, Atomic Transient Recorder, Nature (London) 427, 817 (2004).

[49] E. Goulielmakis, V. S. Yakovlev, A. L. Cavalieri, M. Uiberacker, V. Pervak, A. Apolonski, R. Kienberger, U. Kleineberg, and F. Krausz, Attosecond Control and Measurement: Lightwave Electronics, Science 317, 769 (2007).

[50] A. L. Cavalieri, N. Müller, Th. Uphues, V. S. Yakovlev, A. Baltuska, B. Horvath, B. Schmidt, L. Blümel, R. Holzwarth, S. Hendel, M. Drescher, U. Kleineberg, P. M. Echenique, R. Kienberger, F. Krausz, and U. Heinzmann, Attosecond Spectroscopy in Condensed Matter, Nature (London) 449, 1029 (2007).

[51] X. Zhao, H. Wei, Y. Wu, and C. D. Lin, Phase-Retrieval Algorithm for the Characterization of Broadband Single Attosecond Pulses, Phys. Rev. A 95, 043407 (2017).

[52] S. Pabst and J. M. Dahlström, Eliminating the Dipole Phase in Attosecond Pulse Characterization Using Rydberg Wave Packets, Phys. Rev. A 94, 013411 (2016).

[53] S. Pabst and J. M. Dahlström, Characterizing Attosecond Pulses in the Soft X-Ray Regime, J. Phys. B 50, 104002 (2017).

[54] J. L. Krause, K. J. Schafer, and K. C. Kulander, High-Order Harmonic Generation from Atoms and Ions in the High Intensity Regime, Phys. Rev. Lett. 68, 3535 (1992).

[55] M. V. Frolov, N. L. Manakov, and A. F. Starace, Wavelength Scaling of High-Harmonic Yield: Threshold Phenomena and Bound State Symmetry Dependence, Phys. Rev. Lett. 100, 173001 (2008).

[56] D. Austin and J. Biegert, Strong-Field Approximation for the Wavelength Scaling of High-Harmonic Generation, Phys. Rev. A 86, 023813 (2012).

[57] T. Popmintchev, M.-C. Chen, A. Bahabad, M. Gerrity, P. Sidorenko, O. Cohen, I. P. Christov, M. M. Murnane, and H. C. Kapteyn, Phase Matching of High Harmonic Generation in the Soft and HardX-Ray Regions of the Spectrum, Proc. Natl. Acad. Sci. U.S.A. 106, 10516 (2009).

[58] J. J. Yeh and I. Lindau, Atomic Subshell Photoionization Cross Sections and Asymmetry Parameters: $1 \leq Z \leq 103$, At. Data Nucl. Data Tables 32, 1 (1985).

[59] D. R. Austin and J. Biegert, Attosecond Pulse Shaping Using Partial Phase Matching, New J. Phys. 16, 113011 (2014). 
[60] K. T. Kim, C. M. Kim, M. G. Baik, G. Umesh, and C. H. Nam, Single Sub-50-Attosecond Pulse Generation from Chirp-Compensated Harmonic Radiation Using Material Dispersion, Phys. Rev. A 69, 051805 (2004).

[61] G. Doumy, J. Wheeler, C. Roedig, R. Chirla, P. Agostini, and L. F. Dimauro, Attosecond Synchronization of HighOrder Harmonics from Midinfrared Drivers, Phys. Rev. Lett. 102, 093002 (2009).

[62] Y. Mairesse, A. de Bohan, L. J. Frasinski, H. Merdji, L. C. Dinu, P. Monchicourt, P. Breger, M. Kovačev, R. Taïeb, B. Carré, H. G. Muller, P. Agostini, and P. Salières, Attosecond Synchronization of High-Harmonic Soft X-Rays, Science 302, 1540 (2003).

[63] D. H. Ko, K. T. Kim, and C. H. Nam, Attosecond-Chirp Compensation with Material Dispersion to Produce Near Transform-Limited Attosecond Pulses, J. Phys. B 45, 074015 (2012).

[64] J. Gagnon and V.S. Yakovlev, The Robustness of Attosecond Streaking Measurements, Opt. Express 17, 17678 (2009).

[65] J. Gagnon, E. Goulielmakis, and V.S. Yakovlev, The Accurate FROG Characterization of Attosecond Pulses from Streaking Measurements, Appl. Phys. B 92, 25 (2008).

[66] U. Becker and D. A. Shirley, VUV and Soft X-Ray Photoionization (Springer, Boston, 1996), pp. 135-180.

[67] D. W. Lindle, P. A. Heimann, T. A. Ferrett, P. H. Kobrin, C. M. Truesdale, U. Becker, H. G. Kerkhoff, and D. A.
Shirley, Photoemission from the $3 d$ and $3 p$ Subshells of $\mathrm{Kr}$, Phys. Rev. A 33, 319 (1986).

[68] S. Ricz, R. Sankari, Á. Kövér, M. Jurvansuu, D. Varga, J. Nikkinen, T. Ricsoka, H. Aksela, and S. Aksela, Strong Nondipole Effect Created by Multielectron Correlation in 5s Photoionization of Xenon, Phys. Rev. A 67, 012712 (2003).

[69] A. J. Verhoef, A. V. Mitrofanov, X. T. Nguyen, M. Krikunova, S. Fritzsche, N. M. Kabachnik, M. Drescher, and A. Baltuśka, Time-and-Energy-Resolved Measurement of Auger Cascades Following Kr 3d Excitation by Attosecond Pulses, New J. Phys. 13, 113003 (2011).

[70] J. Jauhiainen, A. Kivimaki, S. Aksela, O. P. Sairanen, and H. Aksela, Auger and Coster-Kronig Decay of the $3 p$ Hole States in Krypton, J. Phys. B 28, 4091 (1995).

[71] V. Jonauskas, S. Kućas, and R. Karazija, Auger Decay of 3p-Ionized Krypton, Phys. Rev. A 84, 053415 (2011).

[72] A. S. Kheifets, Time Delay in Valence-Shell Photoionization of Noble-Gas Atoms, Phys. Rev. A 87, 063404 (2013).

[73] F. Calegari, M. Lucchini, G. Sansone, S. Stagira, C. Vozzi, and M. Nisoli, in Attosecond Physics: Attosecond Measurements and Control of Physical Systems, edited by L. Plaja, R. Torres, and A. Zaïr (Springer, Berlin, 2013), pp. 69-80.

[74] S. M. Teichmann, F. Silva, S. L. Cousin, M. Hemmer, and J. Biegert, 0.5-keV Soft X-Ray Attosecond Continua, Nat. Commun. 7, 11493 (2016).

[75] M. V. Ammosov, N. B. Delone, and V. P. Krainov, Z. Eksp. Teor. Fiz. 91, 2008 (1986) [Sov. Phys. JETP 64, 1191 (1986)]. 